\title{
A Coordinative Solubilizer Method to Fabricate Soft Porous Materials from Insoluble Metal-Organic Polyhedra**
}

\author{
Arnau Carné-Sánchez, * Gavin A. Craig, Patrick Larpent, Vincent Guillerm, Kenji Urayama, \\ Daniel Maspoch, and Shuhei Furukawa*
}

\begin{abstract}
Porous molecular cages have a characteristic processability arising from their solubility, which allows their incorporation into porous materials. Attaining solubility often requires covalently bound functional groups that are unnecessary for porosity and which ultimately occupy free volume in the materials, decreasing their surface areas. Here, a method is described that takes advantage of the coordination bonds in metal-organic polyhedra (MOPs) to render insoluble MOPs soluble by reversibly attaching an alkyl-functionalized ligand. We then use the newly soluble MOPs as monomers for supramolecular polymerization reactions, obtaining permanently porous, amorphous polymers with the shape of colloids and gels, which display increased gas uptake in comparison with materials made with covalently functionalized MOPs.
\end{abstract}

Amorphous, porous materials are attractive for materials processing because their principal functionality, the pore, does not depend on order in the network. ${ }^{[1]}$ At the same time,

[*] Dr. A. Carné-Sánchez, Dr. G. A. Craig, Dr. P. Larpent, Prof. S. Furukawa

Institute for Integrated Cell-Material Sciences (WPI-iCeMS)

Kyoto University

Yoshida, Sakyo-ku, Kyoto 606-8501 (Japan)

E-mail: Shuhei.furukawa@icems.kyoto-u.ac.jp

Dr. A. Carné-Sánchez, Dr. V. Guillerm, Prof. D. Maspoch

Catalan Institute of Nanoscience and Nanotechnology (ICN2), CSIC

The Barcelona Institute of Science and Technology

Campus UAB, Bellaterra, 08193 Barcelona (Spain)

E-mail: arnau.carne@icn2.cat

Prof. K. Urayama

Department of Macromolecular Science and Engineering

Kyoto Institute of Technology

Matsugasaki, Sakyo-ku, Kyoto 606-8585 (Japan)

Prof. D. Maspoch

ICREA

Pg. Lluís Companys 23, 08010 Barcelona (Spain)

Prof. S. Furukawa

Department of Synthetic Chemistry and Biological Chemistry,

Graduate School of Engineering, Kyoto University

Katsura, Nishikyo-ku, Kyoto 615-8510 (Japan)

[**] A previous version of this manuscript has been deposited on a preprint server (https://doi.org/10.26434/chemrxiv.7460618.v1).

(2) Supporting information and the ORCID identification number(s) for

(iD the author(s) of this article can be found under: https://doi.org/10.1002/anie.201901668.

of (c) 2019 The Authors. Published by Wiley-VCH Verlag GmbH \& Co. $\mathrm{KGaA}$. This is an open access article under the terms of the Creative Commons Attribution-NonCommercial License, which permits use, distribution and reproduction in any medium, provided the original work is properly cited and is not used for commercial purposes. this can make it difficult to engineer the size of the pores. One possible approach to control pore size is to use molecular cages such as porous organic cages or metal-organic polyhedra (MOPs) as pre-synthesized building blocks that will retain their known porous structure even in the amorphous state. ${ }^{[2]}$ The processability of molecular cages depends on being able to manipulate them in the solution phase. To ensure solubility, and avoid uncontrolled aggregation into insoluble phases, the cages are often covalently functionalized with bulky side groups. ${ }^{[3]}$ The cost of this approach is that these covalently bound moieties can be detrimental to the gas uptake of the materials. ${ }^{[4]}$

Herein, we show how unfunctionalized, insoluble MOPs can be incorporated into porous, amorphous polymers (Figure 1). We build on our recent study of polymeric
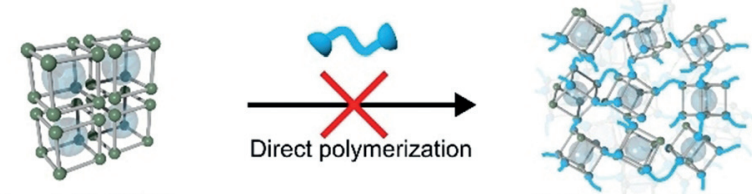

Insoluble MOPs
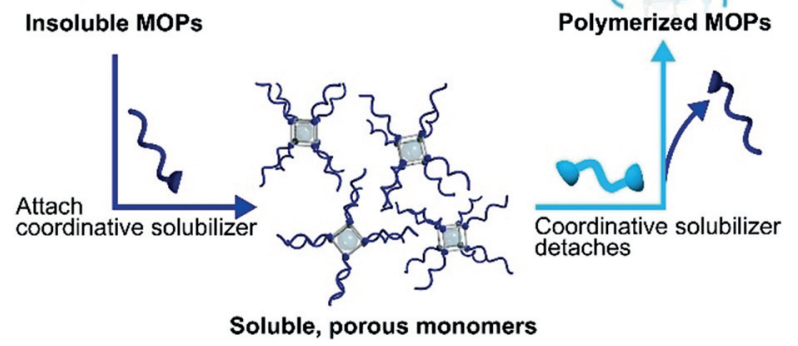

Figure 1. The strategy used in this paper to solubilize MOPs. Assemblies of insoluble MOPs are made soluble by the attachment of a monodentate, coordinative solubilizer. These monodentate ligands can then be replaced with bidentate linkers, driving the assembly of the MOPs into porous polymers.

MOPs (polyMOPs) with permanent microporosity synthesized through coordination-driven supramolecular polymerization, ${ }^{[5]}$ in which the soluble $\mathrm{MOP}\left[\mathrm{Rh}_{2}\left(\mathrm{C}_{12} \text {-bdc }\right)_{2}\right]_{12}$ $\left(\mathbf{C}_{12}\right.$ RhMOP; $\mathrm{C}_{12}$-bdc $^{2-}=5$-dodecoxybenzene-1,3-dicarboxylate $)^{[6]}$ was used as an intrinsically porous monomer. There, the dodecoxy chains that made the MOPs soluble occupied free volume in the final assembly, hindering gas diffusion, and lowering the capacity of the polyMOPs. In this study, we attach a molecule at the periphery of unfunctionalized, insoluble MOPs through labile coordination bonds: a coordinative solubilizer. In the subsequent polymerization reaction, this molecule detaches and is replaced by a bis-mono- 
dentate linker. The obtained polymers show superior gas uptake compared to the covalently functionalized polymers.

First, we chose the cuboctahedral MOP $\left[\mathrm{Rh}_{2}(\mathrm{bdc})_{2}\right]_{12}$ (HRhMOP; $\mathrm{bdc}^{2-}=$ benzene-1,3-dicarboxylate; Supporting Information, Figure S1) because the complex is microporous, presenting one of the highest BET surface areas ${ }^{[7]}$ reported for MOPs $\left(\mathrm{S}_{\mathrm{BET}}=915 \mathrm{~m}^{2} \mathrm{~g}^{-1}\right){ }^{[8]}$ However, HRhMOP is insoluble in organic solvents, impeding its use as a porous monomer. Thus, we exploited the reactivity of the axial site of the dirhodium paddlewheel to coordinate the alkyl-imidazole ligand 1-dodecyl- $1 \mathrm{H}$-imidazole (diz) as a coordinative solubilizer to the external axial site. ${ }^{[9]}$ First, crystals of HRhMOP were dispersed in dichloromethane (DCM), before addition of 15 mol equiv (with respect to HRhMOP) of diz dissolved in DCM. Immediately, the green suspension became a purple solution, suggesting the coordination of diz to HRhMOP (Figure 2). The solvent was removed in vacuo, and the solid

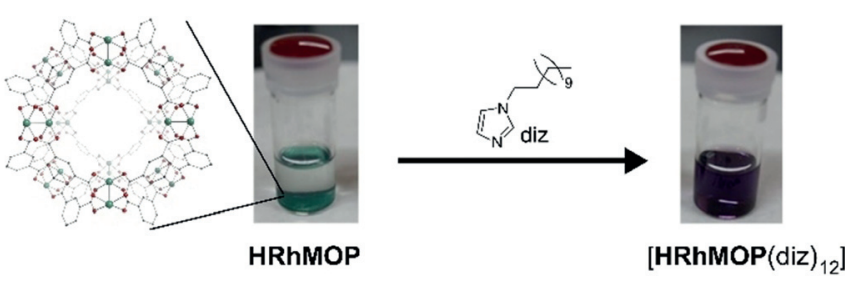

Figure 2. Representation of the molecular structure of HRhMOP, with hydrogen atoms and coordinated solvent molecules omitted for clarity. Photographs of a suspension of HRhMOP in DCM, and the solution of [HRhMOP $(\mathrm{diz})_{12}$ ] obtained following addition of the coordinative solubilizer diz.

residue washed to remove excess diz. The obtained solid was soluble in common organic solvents such as tetrahydrofuran, dimethylformamide (DMF), DCM, and chloroform, allowing its characterization with spectroscopic techniques. Dynamic light scattering (DLS) analysis showed the presence of isolated molecules of an average size of $3.3 \pm 0.5 \mathrm{~nm}$. UV/ Vis spectroscopy revealed that the maximum $\left(\lambda_{\max }\right)$ of the band associated to the dirhodium paddlewheel was centered at $560 \mathrm{~nm}$, corresponding to the coordination of one diz ligand per paddlewheel (Supporting Information, Figure S2). NMR spectroscopy showed the coordination of diz to HRhMOP in solution and was used to determine the molecular formula of the functionalized HRhMOP as $\left[\mathrm{Rh}_{2}(\mathrm{bdc})_{2}(\mathrm{diz})\right]_{12}$ ([HRhMOP( $\left.(\mathrm{diz})_{12}\right]$; Supporting Information, Figure S3).

We tested the reactivity of this soluble, isolated MOP towards the imidazole-based ditopic ligand bix (1,4-bis(imidazol-1-ylmethyl)benzene). A $0.23 \mathrm{~mm}$ DMF solution of [HRhMOP $\left.(\mathrm{diz})_{12}\right]$ was titrated with bix until $12 \mathrm{~mol}$ equiv had been added. While UV/Vis spectroscopy showed no change in $\lambda_{\max }$ (Supporting Information, Figure S4), DLS measurements revealed a self-assembly process: upon addition of 12 mol equiv of bix, the particle size grew from $3.3 \pm$ $0.5 \mathrm{~nm}$ to $44.6 \pm 1.6 \mathrm{~nm}$ (Figure $3 \mathrm{a}$ ). The constant value of $\lambda_{\max }$ throughout self-assembly suggests that any ligand exchange reaction occurs concertedly, with the number of axial sites occupied by imidazole ligands remaining constant at 12 during the process. The colloidal suspension was centrifuged to isolate an amorphous purple solid consisting

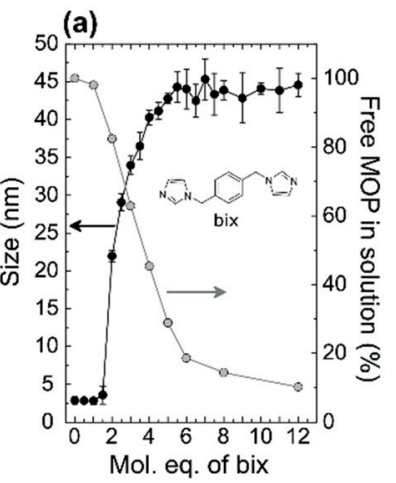

(b)

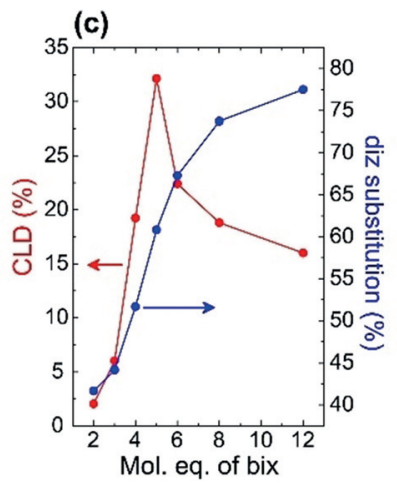

Figure 3. a) In black, the size evolution of the colloids observed during the titration of [HRhMOP $(\mathrm{diz})_{12}$ ] with bix, as determined by DLS measurements $(0.23 \mathrm{~mm}$ in DMF). Error bars correspond to the standard deviation of three repetitions. In gray, the decrease in the free HRhMOP molecules in solution as the particles of CPP grow, determined from the supernatant of the suspensions. b) Representative dark field STEM image of the colloids of CPP obtained after the titration; scale bar: $200 \mathrm{~nm}$. c) In red, evolution of the cross-linking degree (CLD) during particle growth. In blue, the degree of substitution of diz by bix.

of coordination polymer particles (CPP) with an average size of $40 \pm 7 \mathrm{~nm}$ (Figure $3 \mathrm{~b}$ ). The infrared (IR) spectrum of CPP confirmed the presence of HRhMOP units and bix linkers, and also showed a decrease but not a total disappearance of the bands arising from diz (Supporting Information, Figure S5); that is, diz was only partially replaced by bix during polymerization. This was further evidenced by NMR analysis of the acid-digested particles, which displayed peaks from both bix and diz. The relative integrations for the linkers indicated the composition of CPP to be $\left[\mathbf{H R h M O P}(\text { bix })_{8}{ }^{-}\right.$ $(\mathrm{diz})_{2.7}$ (Supporting Information, Figure S6). Because there are 12 paddlewheels in HRhMOP and each paddlewheel only coordinates one $\mathrm{N}$-donor ligand on the exohedral site, it follows that not all of the bix molecules in CPP act as bidentate ligands, as this would limit the maximum number of bix ligands to 6 . We define the cross-linking degree (CLD) as the fraction of bix molecules acting as bidentate ligands [Eq. (1)]. Therefore, the CLD of CPP is $16 \%$.

$\mathrm{CLD}=\frac{\text { bidentate bix }}{\text { total bix }}=\frac{12-\text { diz }- \text { bix }}{\text { bix }} \times 100$

These characterization data are consistent with a selfassembly mechanism entailing ligand exchange between diz 
and bix. However, the growth profile observed by DLS measurements suggests a complex pathway, whereby a threshold quantity of bix is required to begin particle growth. Above 5 mol equiv of bix, particle growth stops, as beyond this point most of the free HRhMOP molecules in solution have been incorporated into the polymers (Figure $3 \mathrm{a}$ ). Although continued addition of bix beyond 5 mol equiv leads to substitution of diz, the bix molecules act as monodentate ligands, decreasing the CLD (Figure $3 \mathrm{c}$ ). This mechanism was found to be independent of the concentration at which polymerization was performed; higher concentrations simply led to larger colloids (average size $247 \pm 70 \mathrm{~nm}$; Supporting Information, Figure S7). More details of the mechanism underlying the self-assembly are provided in the Supporting Information.

As found previously for $\mathbf{C}_{\mathbf{1 2}} \mathbf{R h M O P}$, the self-assembly mechanism could be controlled by changing how bix was added. ${ }^{[5]}$ Thus, 12 mol equiv of bix were added in one portion to a $0.93 \mathrm{mM}$ DMF solution of $\left[\mathbf{H R h M O P}(\operatorname{diz})_{12}\right]$. In the case of $\mathbf{C}_{\mathbf{1 2}} \mathbf{R h M O P}$, this yielded a kinetically trapped, isolated molecule with 12 molecules of bix coordinated to the MOP in a monodentate fashion $\left[\mathbf{C}_{\mathbf{1 2}} \mathbf{R h M O P}(\text { bix })_{12}\right]$. Here the situation is different because the axial sites to be occupied by bix are already filled by diz. The kinetically trapped molecule formed from concerted addition of bix to the solution of [HRhMOP $\left.(\mathrm{diz})_{12}\right]$ was obtained by precipitation with diethyl ether, with analysis revealing the composition of these molecules to be $\left[\mathbf{H R h M O P}(\mathrm{bix})_{9.6}(\mathrm{diz})_{2.4}\right]$ (Supporting Information, Figures S8 and S9). Heating the DMF solution of these molecules at $80^{\circ} \mathrm{C}$ led to the formation of a transparent gel within a few minutes (SCG: supramolecular colloidal gel), with gelation also occurring at room temperature, albeit at a slower rate (Figure 4). Gel-like behavior was confirmed by rheological measurements, which determined a frequencyindependent storage modulus $E^{\prime}$ of about $4 \mathrm{kPa}$, which is one order of magnitude higher than the loss modulus. The composition of SCG was found to be $\left[\mathbf{H R h M O P}(\text { bix })_{9.5^{-}}\right.$ $\left.(\mathrm{diz})_{1.3}\right]$, corresponding to a CLD of $12 \%$ (Supporting Information, Figure S10). The decrease in diz content upon assembly of the gel while the bix content is retained demonstrates that the self-assembly process is driven by the reactivity of the monodentate bix molecules replacing diz to crosslink the MOPs. Through solvent exchange with acetone followed by supercritical $\mathrm{CO}_{2}$ drying, the gel was converted to a supramolecular aerogel (SAG) consisting of fused nanoparticles with an average size of $29.8 \mathrm{~nm}$ (Figure 4; Supporting Information, Figure S11).

The aim of these assembly strategies was to demonstrate that an otherwise insoluble MOP could be incorporated into amorphous, supramolecular polymers with permanent microporosity. For these types of polymers, it can be difficult to distinguish between the contributions of intrinsic porosity (arising from the cavity of the MOP) and extrinsic porosity (arising from the free volume between MOPs or particles). To determine the impact of the intrinsic microporosity of HRhMOP on the overall porosity of CPP and SAG, we performed Ar adsorption measurements at $87 \mathrm{~K}$. The low dipole moment of Ar prevents any chemical interaction between the cavity walls and the gas, providing higher quality physisorption measurements. ${ }^{[10]}$ At $87 \mathrm{~K}$, HRhMOP, CPP, (a)

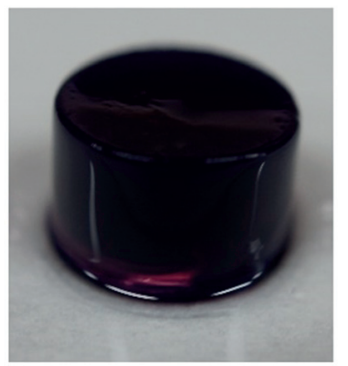

Supramolecular colloidal gel (SCG)

(c)

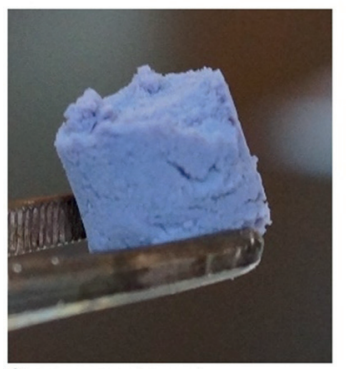

Supramolecular

aerogel (SAG) (b)

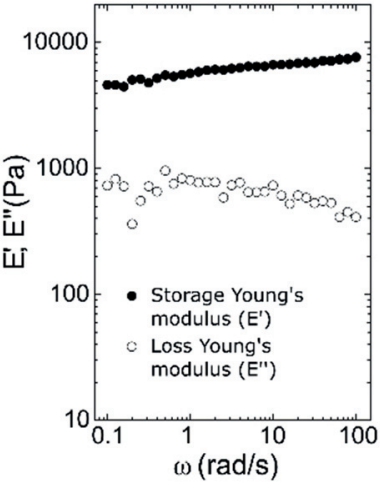

(d)

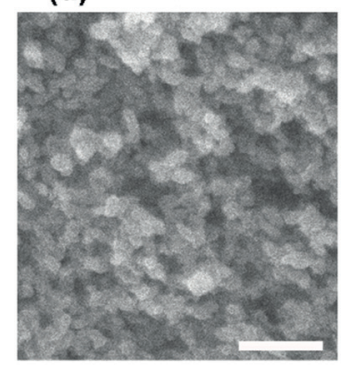

Figure 4. a) Photograph of the supramolecular colloidal gel SCG. b) Storage and loss moduli of SCG as a function of angular frequency. c) Photograph of the supramolecular aerogel SAG obtained after drying SCG with supercritical $\mathrm{CO}_{2}$. d) Representative FESEM image of SAG; scale bar: $200 \mathrm{~nm}$.

(a)

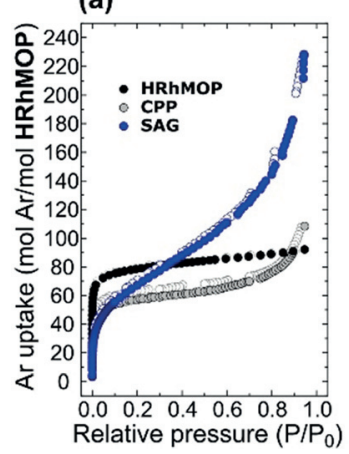

(b)

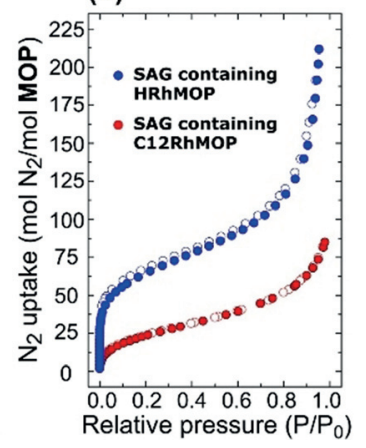

Figure 5. a) Ar adsorption isotherm at $87 \mathrm{~K}$ for HRhMOP (black), CPP (gray), and SAG (blue). b) Comparison of the $\mathrm{N}_{2}$ uptake at $77 \mathrm{~K}$ for the supramolecular aerogels containing HRhMOP and the previously reported $\mathbf{C}_{12}$ RhMOP. Closed and open symbols correspond to the adsorption and desorption branches, respectively.

and SAG all show sharp uptake of Ar at low pressures, characteristic of microporous materials (Figure 5; for volumetric data see the Supporting Information, Figure S12). The pore size distribution (PSD) demonstrates that all of the samples have pores in the microporous range, in good agreement with the dimensions of the cuboctahedral cage (Supporting Information, Figure S13). The shaping process and polymer morphology is important: while for CPP $54 \%$ of the porosity arises from the micropores, SAG presents a much 
wider PSD, where the microporosity accounts for $21 \%$ of its porosity. Furthermore, SAG displays interstitial voids between the spheres, as shown by increased gas uptake in the higher-pressure region. Comparison of the $\mathrm{N}_{2}$ sorption isotherm at $77 \mathrm{~K}$ of the supramolecular aerogel containing HRhMOP with that of the supramolecular aerogel containing $\mathbf{C}_{\mathbf{1 2}} \mathbf{R h M O P}\left(\left[\mathrm{Rh}_{2}\left(\mathrm{C}_{12} \text {-bdc }\right)_{2}\right]_{12}\right.$, where $\mathrm{C}_{12}$-bdc $^{2-}=5$-dodecoxybenzene-1,3-dicarboxylate) emphasizes the detrimental effect of the covalent functionality on gas uptake: at $P / P_{0}$ $\approx 0.01$, the $\mathbf{C}_{\mathbf{1 2}} \mathbf{R h M O P}$ aerogel adsorbs 8 moles of gas per mole of MOP $\left(13.7 \mathrm{~cm}^{3} \mathrm{~g}^{-1}\right)$, while the HRhMOP-based aerogel adsorbs 38.9 moles of $\mathrm{N}_{2}$ per mole of MOP $\left(97.3 \mathrm{~cm}^{3} \mathrm{~g}^{-1}\right)$. Both polymers show increased adsorption of $\mathrm{N}_{2}$ at higher relative pressures arising from macropores in the polymeric assembly, with uptakes of 73.7 moles $\left(125.5 \mathrm{~cm}^{3} \mathrm{~g}^{-1}\right)$ and 202.0 moles $\left(504.0 \mathrm{~cm}^{3} \mathrm{~g}^{-1}\right)$ of $\mathrm{N}_{2}$ at $P / P_{0}$ $\approx 0.95$ for the aerogels based on $\mathbf{C}_{\mathbf{1 2}}$ RhMOP and HRhMOP, respectively.

In summary, we have demonstrated a method to concatenate insoluble MOP molecules into amorphous supramolecular polymers. By reversibly attaching a coordinative solubilizer to the MOPs, the need to covalently functionalize MOPs with bulky moieties to ensure their solubility and, hence, their processability, was avoided. This method could be used in general to incorporate insoluble metal complexes into coordination networks, including metal-organic frameworks (MOFs).

\section{Acknowledgements}

A.C.-S., G.A.C., and P.L. thank the Japan Society for the Promotion of Science (JSPS) for Post-Doctoral Fellowships. This work was supported by JSPS KAKENHI grant number 18H01995 (Kiban B) and 17H05367 (Coordination Asymmetry). This work was also supported by the Spanish MINECO (projects PN MAT2015-65354-C2-1-R), the Catalan AGAUR (project 2014 SGR 80), and the ERC under the EU-FP7 (ERC-Co 615954). It was also funded by the CERCA Program/Generalitat de Catalunya. iCeMS is supported by the World Premier International Research Initiative (WPI), MEXT, Japan. ICN2 is supported by the Severo Ochoa program from the Spanish MINECO (Grant No. SEV-20170706). A.C.S. also thanks the Spanish MINECO for a Juan de la Cierva fellowship (IJCI-2016-29802 and FJCI-2017-31598).

\section{Conflict of interest}

The authors declare no conflict of interest.

Keywords: coordination cages - metal-organic polyhedra · microporous materials · polymers · supramolecular chemistry

How to cite: Angew. Chem. Int. Ed. 2019, 58, 6347-6350

Angew. Chem. 2019, 131, 6413-6416

[1] a) P. M. Budd, B. S. Ghanem, S. Makhseed, N. B. McKeown, K. J. Msayib, C. E. Tattershall, Chem. Commun. 2004, 230; b) J.-
X. Jiang, F. Su, A. Trewin, C. D. Wood, N. L. Campbell, H. Niu, C. Dickinson, A. Y. Ganin, M. J. Rosseinsky, Y. Z. Khimyak, A. I. Cooper, Angew. Chem. Int. Ed. 2007, 46, 8574; Angew. Chem. 2007, 119, 8728; c) Y. Zhao, S.-Y. Lee, N. Becknell, O. M. Yaghi, C. A. Angell, J. Am. Chem. Soc. 2016, 138, 10818; d) R. Gaillac, P. Pullumbi, K. A. Beyer, K. W. Chapman, D. A. Keen, T. D. Bennett, F.-X. Coudert, Nat. Mater. 2017, 16, 1149.

[2] a) J.-R. Li, H.-C. Zhou, Angew. Chem. Int. Ed. 2009, 48, 8465; Angew. Chem. 2009, 121, 8617; b) S. Jiang, J. T. A. Jones, T. Hasell, C. E. Blythe, D. J. Adams, A. Trewin, A. I. Cooper, Nat. Commun. 2011, 2, 207; c) J. Tian, S. Ma, P. K. Thallapally, D. Fowler, B. P. McGrail, J. L. Atwood, Chem. Commun. 2011, 47, 7626; d) Y. Jin, B. A. Voss, A. Jin, H. Long, R. D. Noble, W. Zhang, J. Am. Chem. Soc. 2011, 133, 6650; e) J. M. Teo, C. J. Coghlan, J. D. Evans, E. Tsivion, M. Head-Gordon, C. J. Sumby, C. J. Doonan, Chem. Commun. 2016, 52, 276; f) D. Nam, J. Huh, J. Lee, J. H. Kwak, H. Y. Jeong, K. Choi, W. Choe, Chem. Sci. 2017, 8,7765 .

[3] a) H. Furukawa, J. Kim, K. E. Plass, O. M. Yaghi, J. Am. Chem. Soc. 2006, 128, 8398; b) T. Tozawa, J. T. A. Jones, S. I. Swamy, S. Jiang, D. J. Adams, S. Shakespeare, R. Clowes, D. Bradshaw, T. Hasell, S. Y. Chong, C. Tang, S. Thompson, J. Parker, A. Trewin, J. Bacsa, A. M. Z. Slawin, A. Steiner, A. I. Cooper, Nat. Mater. 2009, 8, 973; c) J. Tian, P. K. Thallapally, S. J. Dalgarno, P. B. McGrail, J. L. Atwood, Angew. Chem. Int. Ed. 2009, 48, 5492; Angew. Chem. 2009, 121, 5600; d) J.-R. Li, H.-C. Zhou, Nat. Chem. 2010, 2, 893; e) G. Zhang, O. Presly, F. White, I. M. Oppel, M. Mastalerz, Angew. Chem. Int. Ed. 2014, 53, 1516; Angew. Chem. 2014, 126, 1542; f) M. Kitchin, J. Teo, K. Konstas, C. H. Lau, C. J. Sumby, A. W. Thornton, C. J. Doonan, M. R. Hill, J. Mater. Chem. A 2015, 3, 15241; g) V. Brega, M. Zeller, Y. He, H. Peter Lu, J. K. Klosterman, Chem. Commun. 2015, 51, 5077; h) A. Burgun, P. Valente, J. D. Evans, D. M. Huang, C. J. Sumby, C. J. Doonan, Chem. Commun. 2016, 52, 8850; i) G. Lal, M. Derakhshandeh, F. Akhtar, D. M. Spasyuk, J.-B. Lin, M. Trifkovic, G. K. H. Shimizu, J. Am. Chem. Soc. 2019, 141, 1045.

[4] a) M. W. Schneider, I. M. Oppel, H. Ott, L. G. Lechner, H.-J. S. Hauswald, R. Stoll, M. Mastalerz, Chem. Eur. J. 2012, 18, 836; b) E. V. Perez, K. J. Balkus, J. P. Ferraris, I. H. Musselman, J. Membr. Sci. 2014, 463, 82; c) T.-H. Chen, L. Wang, J. V. Trueblood, V. H. Grassian, S. M. Cohen, J. Am. Chem. Soc. 2016, 138, 9646; d) P. S. Reiss, M. A. Little, V. Santolini, S. Y. Chong, T. Hasell, K. E. Jelfs, M. E. Briggs, A. I. Cooper, Chem. Eur. J. 2016, 22, 16547.

[5] A. Carné-Sánchez, G. A. Craig, P. Larpent, T. Hirose, M. Higuchi, S. Kitagawa, K. Matsuda, K. Urayama, S. Furukawa, Nat. Commun. 2018, 9, 2506.

[6] R. Kawano, N. Horike, Y. Hijikata, M. Kondo, A. CarnéSánchez, P. Larpent, S. Ikemura, T. Osaki, K. Kamiya, S. Kitagawa, S. Takeuchi, S. Furukawa, Chem 2017, 2, 393.

[7] C. A. Rowland, G. R. Lorzing, E. J. Gosselin, B. A. Trump, G. P. A. Yap, C. M. Brown, E. D. Bloch, J. Am. Chem. Soc. 2018, 140, 11153.

[8] S. Furukawa, N. Horike, M. Kondo, Y. Hijikata, A. CarnéSánchez, P. Larpent, N. Louvain, S. Diring, H. Sato, R. Matsuda, R. Kawano, S. Kitagawa, Inorg. Chem. 2016, 55, 10843.

[9] A. Carné-Sánchez, J. Albalad, T. Grancha, I. Imaz, J. Juanhuix, P. Larpent, S. Furukawa, D. Maspoch, J. Am. Chem. Soc. 2019, 141, 4094.

[10] K. A. Cychosz, R. Guillet-Nicolas, J. García-Martínez, M. Thommes, Chem. Soc. Rev. 2017, 46, 389.

Manuscript received: February 7, 2019

Accepted manuscript online: March 7, 2019

Version of record online: April 2, 2019 